\title{
Preventative Psychosocial Interventions Following Traumatic Physical Injury: Who, What, When?
}

\author{
Malcolm MacLachlan ${ }^{*}$, , Mary De Silva ${ }^{2}$, Declan Devane ${ }^{3}$ and Vikram Patel ${ }^{2}$ \\ ${ }^{1}$ Centre for Global Health and School of Psychology, Trinity College, University of Dublin, Dublin, Ireland \\ ${ }^{2}$ Nutrition \& Public Health Intervention Research Unit, London School of Hygiene \& Tropical Medicine, London, UK \\ ${ }^{3}$ School of Nursing and Midwifery, National University of Ireland Galway, Galway, Ireland
}

\begin{abstract}
Kornør et al. [1] reviewed the results of studies comparing early trauma-focused cognitive-behavioural therapy (TFCBT) with supportive counselling (SC) in people identified as being at risk of developing traumatic stress related symptoms. They suggest that their results provide some evidence for the relative benefit of TFCBT in preventing chronic post-traumatic stress disorder (PTSD), and related symptoms. We question the extent to which this conclusion applies to the subgroup of people who have experienced traumatic physical injuries. Our systematic review of the efficacy of psychosocial interventions for preventing the onset of disability in people who had experienced traumatic physical injuries emphasizes the equivocal nature of current research, notes some negative outcomes and cautions for very careful monitoring of such interventions should they be used. There is a need for further high quality research exploring the optimal timing and nature of such interventions, as well as who is most likely to benefit from them.
\end{abstract}

Kornør et al. [1] sought to evaluate the effectiveness of trauma-focused cognitive behaviour therapy (TFCBT) in preventing chronic Post-Traumatic Stress Disorder (PTSD), anxiety and depression. They included randomised controlled trials (RCTs) published in peer reviewed journals; people with diagnosed symptoms of Acute Stress Disorder (ASD) or symptoms of PTSD; those who had received TFCBT initiated within three months of the identified traumatic event; a non-pharmacological comparison intervention; and where outcomes were measured at followup of a minimum of one month after treatment. Seven papers from an initial search of 1438 studies met these criteria.

While Kornør et al. [1] report significant effects across these studies at 3-6 month follow-up, such effects were not found at 9 months, or 3-4 years post-treatment. Although trends supported the relative efficacy of TFCBT, the results were inconclusive. However, it is not clear the extent to which these interventions can be considered as preventative, rather than therapeutic. Given that an inclusion criterion was symptoms of Acute Stress Disorder (ASD) or PTSD, it may be argued that the interventions sought to avoid the worsening of symptoms, rather than to prevent them. Furthermore, while Bryant et al. [2] recently sought to evaluate the efficacy of exposure $v s$ cognitive restructng for trauma survivors with acute stress disorder (ASD), their intervention was targeted at people who already had ASD, with a view to 'preventing' them subsequently developing chronic posttraumatic stress disorder (PTSD).

Roberts et al. [3] conducted a systematic review of RCTs of multiple-session psychological treatments that aimed at

*Address correspondence to this author at the Centre for Global Health and School of Psychology, Trinity College Dublin 2, Ireland;

Tel: +353 18961452; E-mail: malcolm.maclachlan@tcd.ie preventing or reducing traumatic stress symptoms in individuals within 3 months of exposure to a traumatic event. For individuals exposed to a traumatic event, irrespective of their symptoms, there was no significant difference between any intervention and usual care. Considering the data in terms of treatment of traumatic stress symptoms, irrespective of diagnosis, trauma-focused cognitive-behavioral therapy (CBT) was more effective than being on a waiting list or receiving 'supportive counselling' in reducing these symptoms.

It is possible that the efficacy of psychosocial interventions may be influenced by the nature of the trauma experienced - specifically whether or not it involves physical injury - and the purpose of the intervention - whether it is preventative or curative. In a recently published review conducted by De Silva et al. [4] we sought to evaluate the effectiveness of psychosocial interventions for the primary prevention of disability following traumatic physical injury. We distinguished between interventions that were purely preventative and those that were curative. Preventative interventions are given to prevent difficulties arising, while curative interventions are given to treat a difficulty that has already arisen. For instance, someone who has a leg amputated may receive interventions that motivate them to ambulate, make appropriate use of their prosthesis, secure networks of social support and identify when they are thinking negatively. Such interventions may therefore reduce the extent to which they experience physical activity limitations and mental health problems. More generally, we argue that physical injury may result in impairment of physical functioning, and that the way in which people respond to such impairment influences the degree of disability associated with the injury. An individual's ability to cope with physical impairment, as well as their broader social situation, therefore offers opportunities to reduce the 
extent to which physical injury may result in disability. Psychosocial interventions may help by providing people with psychological and social resources that assist their coping with physical impairment, thus preventing the development of physical, mental and social disability. We therefore sought to understand the consequences of traumatic physical injury in terms that were broader than the occurrence or non-occurrence of ASD or PTSD symptoms (see De Silva et al. [4] for further details) and more in keeping with the ICF (World Health Organisation International Classification of Functioning, Disability and Health) classification of disability as arising from physical or functional impairments involving an organ or body part, activity limitations involving the whole body or person, and participation restrictions involving the person in his or her environment [5]. Such a classification system, endorsed by all 191 WHO member states in 2001, shifts the focuses of disability "from cause to impact", envisaging disability as a composite of physical, psychological and social difficulties, rather than simply as a dichotomous variable describing the presence or absence of physical or mental 'deficits'.

For the purposes of our review we defined psychosocial interventions as interventions that have their primary mode of action through psychological or social processes. Such interventions include, for instance, direct therapeutic work, health education and social support. Our inclusion criteria were RCTs that considered one or more psychosocial intervention for the prevention of physical disability, mental health problems or reduced social functioning as a result of traumatic physical injury. Trials excluded people who had traumatic brain injury, sexual assault, and people without traumatic physical injury. We included interventions administered by any health professional or lay person, and in any form, for example individual or group therapy, over the telephone, or in the form of written material. We included psychosocial interventions when they were compared with usual care, pharmacological interventions, other treatments for mental health problems and physical interventions. We excluded interventions designed to be therapeutic rather than preventative and studies that purposively selected participants on the basis of disability (including mental health) related problems. From an initial search that identified 1420 papers, 5 met the inclusion criteria.

Two studies explored the preventative effects of Cognitive-Behavioural Therapy (CBT), one Interpersonal Therapy (IT), one Collaborative Care (CC) and one the effects of a Self-Help Information Booklet (SHIB). Burns et al. [6] studied 170 patients who had undergone surgery for hip fracture and who had been classified as not at risk from depression (score of six or less on the Geriatric Depression Scale). Intervention participants received up to seven sessions of CBT while the control group received treatment as usual. There were no significant differences in any of the outcomes (depression, mobility, pain, physical illness, functioning, or length of hospital stay) at follow-up of six weeks, three months or six months.

Pirente et al. [7] followed up 92 severely injured trauma patients who either received up to eight sessions of individual CBT, or treatment as usual. There were significant between group differences at baseline with more participants with anxiety and depression in the intervention compared to the control group. However, there were no significant between-group differences at 6 or 12 months follow-up on any of the outcomes (health related quality of life, depression or anxiety),

Holmes et al. [8] compared 90 major physical trauma patients who had either an average of 5.9 Interpersonal Counselling (IPC) sessions, or treatment as usual. While there was a high rate of drop-out from the intervention group $(47.1 \%)$, no significant between group differences were found for any of the outcomes (depression, anxiety, PTSD, alcohol and substance abuse, any psychiatric disorder and physical functioning).

Zatzick et al. [9] compared 34 injured patients admitted to a trauma centre who received either collaborative care (comprising a personally assigned trauma support specialist and a brief psycho-educational intervention targeting PTSD) or treatment as usual. While at one month follow-up, in intention to treat analyses, the intervention group had significantly decreased PTSD and borderline significant decreased depression symptoms when compared to the control group, at four months the intervention groups' symptoms had significantly increased relative to the control groups for, both PTSD and depression. There were no significant between group differences at either one or four months for the other outcomes (drinking to the point of intoxication and functional limitations).

Turpin et al. [10] gave 291 Accident and Emergency patients who had sustained a physical injury either a selfhelp information booklet (SHIB) six to eight weeks after hospital attendance, or a letter without the booklet. The SHIB described and normalised common physiological, psychological and behavioural reactions to traumatic injury and gave advice on non-avoidance, emotional coping and seeking further help. Only 10\% (291/2818) of those eligible agreed to participate. For the $34 \%$ of those who were successfully followed-up at six months, there were no significant differences between groups in anxiety or PTSD symptoms. However, at three months follow-up there was a statistically boarder-line greater reduction in PTSD caseness in the control than the intervention group $(\mathrm{p}=0.06)$, and in an intention to treat analysis at six months there was a statistically boarder-line higher proportion of depressed patients in the intervention rather than the control group ( $18 \%$ vs $7 \%, \mathrm{p}=0.054)$.

Mental health status was the only disability outcome affected by any intervention in our review. In two trials the psychosocial intervention had a detrimental effect on the mental health status of patients. Our findings cannot therefore be taken as supporting the provision of psychosocial intervention to prevent the occurrence of mental health problems associated with traumatic physical injury.

Both the Kornør et al. [1] and De Silva et al. [4] systematic reviews highlight the lack of fully reported and rigorously designed intervention studies, and so the findings of each of these reviews are necessarily tentative. It seems unclear who is most likely, if anyone, to benefit from preventative (as opposed to curative) psychosocial interventions for people who have received traumatic physical injuries; what sort of interventions might be most 
successful, and when is the optimum time to intervene to prevent disability, including mental health problems. While the philosophy of preventative interventions is attractive, more research needs to be undertaken to identify if, when and for whom, they may be beneficial. Given the lack of evidence for the effectiveness of psychological debriefing following trauma and the suggestion that it may actually have harmful effects $[11,12]$, we stress that any such research will require very close monitoring with protocols that can quickly and easily detect worsening mental health among participants.

\section{REFERENCES}

[1] Kornør H, Winje D, Ekeberg Ø, et al. Early trauma-focused cognitive-behaviour therapy to prevent chronic post-traumatic stress disorder and related symptoms: A systematic review and meta-analysis. BMC Psychiatry 2008; 8: 81.

[2] Bryant RA, Mastrodomenico J, Felmingham KL, et al. Treatment of acute stress disorder: a randomized controlled trial. ARCH Gen Psychiatry 2008; 65: 659-67.

[3] Roberts NP, Kitchiner NJ, Kenardy J, Bisson JI. Systematic review and meta-analysis of multiple-session early interventions following traumatic events. Am J Psychiatry 2009; 166: 293-301.

[4] De Silva M, MacLachlan M, Devane D, et al. Psychosocial interventions for the primary prevention of disability following traumatic physical injury. Cochrane Database Syst Rev 2009; (In press).
[5] World Health Organisation International Classification of Functioning, Disability and Health (www.who.int/classification/icf/ in/downloaded 1 July 2009)

[6] Burns A, Banerjee S, Morris J, et al. Treatment and prevention of depression after surgery for hip fracture in older people: randomized, controlled trials. J Am Ger Soc 2007; 55: 75-80.

[7] Pirente N, Blum C, Wortberg S, et al. Quality of life after multiple trauma: the effect of early onset psychotherapy on quality of life in trauma patients. Langenbecks Arch Surg 2007; 392: 739-45.

[8] Holmes A, Hodgins G, Adey S, et al. Trial of interpersonal counselling after major physical trauma. Aus NZ J Psychiatry 2007; 41: 926-33

[9] Zatzick DF, Roy-Byrne P, Russo JE, et al. Collaborative interventions for physically injured trauma survivors: a pilot randomized effectiveness trial. Gen Hosp Psychiatry 2001; 23: $114-23$

[10] Turpin G, Downes M, Mason S. Effectiveness of giving self-help information acute traumatic injury: a randomised controlled trial. Br J Psychiatry 2005; 187: 76-82.

[11] Rose S, Bisson J, Churchill R, Wessely S. Psychological debriefing for preventing post traumatic stress disorder (PTSD). Cochrane Database Sys Rev 2002, Issue 2. Art. No.: CD000560. DOI: 10.1002/14651858.CD000560.

[12] National Institute of Clinical Excellence (NICE). Post-traumatic Stress Disorder - The management of PTSD in adults and children in primary and secondary care. National Clinical Practice Guideline. London: Gaskell and the British Psychological Society, 2005 . 